\title{
Integral Field Spectroscopic Data Reduction Method for High Resolution Infrared Observation
}

\author{
Sungho Lee ${ }^{1}$, Soojong $\mathrm{Pak}^{2 \dagger}$, and Minho Choi ${ }^{1}$
}

${ }^{1}$ Korea Astronomy and Space Science Institute, Daejeon 305-348, Korea

${ }^{2}$ School of Space Research, Kyung Hee Univeristy, 1 Seocheon-dong, Giheung-gu, Yongin-si, Gyeonggi-do 446-701, Korea

We introduce a technical approach for reducing three-dimensional infrared (IR) spectroscopic data generated by integral field spectroscopy or slit-scanning observations. The first part of data reduction using IRAF presents a guideline for processing spectral images from long-slit IR spectroscopy. Multichannel image reconstruction, Image Analysis and Display (MIRIAD) is used in the later part to construct and analyze the data cubes which contain spatial and kinematic information of the objects. This technic has been applied to a sample data set of diffuse 2.1218 $\mu$ m $\mathrm{H}_{2} 1$ - 0 S(1) emission features observed by slit-scanning around Sgr A East in the Galactic center. Details of image processing for the high-dispersion infrared data are described to suggest a sequence of contamination cleaning and distortion correction. Practical solutions for handling data cubes are presented for survey observations with various configurations of slit positioning.

Keywords: image processing techniques, spectroscopic

\section{INTRODUCTION}

Imaging spectroscopy or integral field spectroscopy provides spectral information at every angular position in a field. The resulting product is a three-dimensional (3D) data cube which comprises spatial information of the observed object in two dimensions and spectral information in the other dimension. The history of imaging spectroscopy in the near-infrared (NIR) is relatively short, but its potential of applying to various astrophysical issues had already been proven (see Weitzel et al. 1996 and references therein). For example, this observing technic is powerful to build emission line maps of extended objects since both the spectral lines and continuum are observed together at the same time, in the same spectroscopic format, and at each position, and the continuum is effectively subtracted using adjacent pixels along the spectral axis of the data cube. It is superior to the imaging technic using narrow-band filters particularly in such a crowded region as the Galactic center or massive starforming regions where seeing variation prohibits accurate continuum subtraction (Burton \& Allen 1992, Lee et al. 2005).

To meet increasing demand in the community, a number of integral field NIR spectrographs with integral field units or image slicer units have been developed and currently in operation; including Gemini/NIFS (McGregor et al. 2003), VLT/SINFONI (Eisenhauer et al. 2003), and Keck/OSIRIS (Larkin et al. 2006). Slit scanning (stepping the slit position of a normal spectrograph across the target) is another method for imaging spectroscopy. Even though this technique is affected by seeing variations, we can observe large extended objects with long-slits or take very high spectral resolution spectra. We plan to apply this observing technique to cross-dispersed high resolution IR spectrographs such as Immersion GRating INfrared Spectrograph (IGRINS; Yuk et al. 2010) and Giant Magellan Telescope Near-Infrared Spectrograph (GMTNIRS; (c) This is an Open Access article distributed under the terms of the Creative Commons Attribution Non-Commercial License (http://creativecommons.org/licenses/by-nc/3.0/) which permits unrestricted non-commercial use, distribution, and reproduction in any medium, provided the original work is properly cited.
Received Oct 26, 2010 Revised Nov 10, 2010 Accepted Nov 17, 2010 ${ }^{\dagger}$ Corresponding Author

E-mail: soojong@khu.ac.kr

Tel: +82-31-201-3813 Fax: +82-31-206-2470 
Lee et al. 2010).

The advantages of high dispersion spectrographs in the integral field modes enable us to study kinematics. For example, Lee et al. (2008) surveyed the circumference of Sgr A East in the Galactic center using the slit scanning technic in $\mathrm{H}_{2} 1-0 S(1)(\lambda=2.1218 \mu \mathrm{m})$ line emission at a high resolving power $(\lambda / \Delta \lambda \simeq 18000)$. This remnant of an energetic explosion is colliding with the ambient material, and line profiles observed toward the shocked gas show its high velocity expanding motion (Lee et al. 2003). Thus Lee et al. (2008) chose high dispersion echelle for the spectroscopic configuration in their observations. The resulting data cube contains information of both structure and kinematics of the shocked $\mathrm{H}_{2}$ gas with high-resolution line profiles at every spatial position. The data in the cube are continuous in the spatial dimensions as well as in the spectral dimension, i.e., along the velocity axis.

A conventional method of reducing the integral field observations is simply stacking two-dimensional (2D) spectral images into a data cube. For example, Burton \& Allen (1992) and Lee et al. (2005) made use of only small portion of their data cubes along the spectral dimension; several slice images in some emission lines. In Lee et al. (2008), however, a unified data cube is required to investigate the entire survey region as a single system, since position and orientation of the slit in their observations changes along the circular boundary of Sgr A East. This type of slit-scanning observation at a high spectral resolution with a complicated survey scheme requires a new technic for data reduction.

In this paper, we introduce the sample data acquired by Lee et al. (2008) in Section 2, and describe in detail the process of data reduction for IR spectroscopy in Section 3. Section 4 presents a new technic for constructing a data cube.

\section{OBSERVATIONS AND DATA}

Lee et al. (2008) surveyed the boundary region of Sgr A East in the $\mathrm{H}_{2}$ 1-0 $S(1)$ emission line which is radiated from shocked gas in the surrounding molecular clouds. They used the Cooled Grating Spectrometer 4 (CGS4; Mountain et al. 1990) at the 3.8 m United Kingdom Infrared Telescope (UKIRT) to observe the $\mathrm{H}_{2}$ line profiles with an echelle grating and a 0.83 arcsec slit width. By the advantage of the long ( $\sim 90 \mathrm{arcsec})$ slit length of CGS4, Lee et al. (2008) were able to scan the large area around Sgr A East with such a high spectral resolution.
Slit scanning observations were implemented in 3 arcsec steps. At the same time, the telescope was nodded between object and blank sky positions every $20 \mathrm{~min}$ utes to subtract background and telluric $\mathrm{OH}$ line emission. Fifty-six slit positions in total were observed over 11 fields around Sgr A East. The slit position angle was various from $-90^{\circ}$ to $+40^{\circ}$ (from north to east). HR 6496 and BS 6310 were observed as standard stars, which are used for flux calibration and distortion correction. More details on the observations can be found in Lee et al. (2008).

\section{REDUCTION OF SPECTRAL IMAGE DATA}

Basic data reduction steps, including bad-pixel-mask correction and flat-fielding (using an internal blackbody lamp), were accomplished by the automated Observatory Reduction and Acquisition Control (ORAC) pipeline at UKIRT. IRAF was used for the remaining part of the reduction process for $2 \mathrm{D}$ (wavelength and position) spectral images at individual slit positions. Stacking and making mosaic of those 2D spectral images into a 3D data cube and the later parts of reduction and analysis thereafter are implemented using Multichannel Image Reconstruction, Image Analysis and Display (MIRIAD; Sault et al. 1995, Hoffman et al. 1996). Flux calibration is

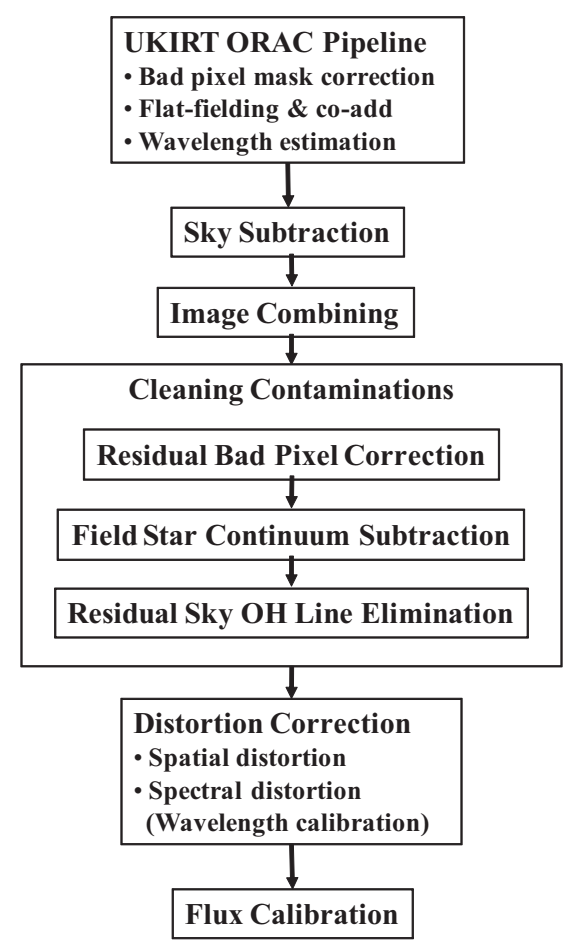

Fig. 1. Reduction sequence of the 2D spectral image data using IRAF. 
not included in this paper but described in detail in Lee $\&$ Pak (2006).

Sequence of data reduction of 2D spectral images using IRAF is shown in Fig. 1. Some steps in the sequence will be described in more detail in the following subsections. This reduction sequence can be modified alternatively depending on observing techniques. For example, if target position was jittered, distortion correction should be done before un-jittering (aligning) the images to be combined. Otherwise the distortions would spread out and become uncorrectable.

\subsection{Cleaning Contaminations}

The wavelength range of $12.5 \mu \mathrm{m}$ is crowded with a dense forest of telluric $\mathrm{OH}$ lines. These emission lines come from a thin layer of the atmosphere at an altitude of about $90 \mathrm{~km}$, and the strength of emission can vary rapidly; by a factor of two or more in half an hour duration (McLean 1997). There are also very strong OH lines near to our objective $\mathrm{H}_{2}$ lines; stronger by orders of magnitude and as close as a few hundred $\mathrm{km} \mathrm{s}^{-1}$ apart. $\mathrm{Sky} \mathrm{OH}$ lines are not subtracted perfectly because of their nature of rapid variations. The high spectral resolving power of CGS4 enables the target $\mathrm{H}_{2}$ emission line to be separated from the brightest residual $\mathrm{OH}$ lines. However, in our case, we found that velocity dispersions of the observed $\mathrm{H}_{2}$ lines are so large that wings of the $\mathrm{H}_{2} 10 \mathrm{~S}(1)$ lines in some spectral images are slightly overlapped with the nearest $\mathrm{OH}$ line (at $\lambda=2.1232424 \mu \mathrm{m}$ which is $\sim 200 \mathrm{~km} \mathrm{~s}^{-1}$ apart from the $\mathrm{H}_{2}$ line; Rousselot et al. 2000).

By subtracting a blank sky image from an object image, the background signals including telluric $\mathrm{OH}$ lines are diminished. Fig. 2 shows the image before (top figure) and after (bottom figure) the subtractions. Vertical lines are telluric $\mathrm{OH}$ lines and horizontal lines are stellar continua. After subtracting the blank sky image, diffuse $\mathrm{H}_{2}$ emission becomes visible in the central part of the image. Since the background and strong $\mathrm{OH}$ lines are diminished, stellar continua look more prominent. Dark horizontal lines in upper part of the bottom figure are the scars due to the stellar continua in the sky image. Note also that the number of serious bad pixels is obviously decreased but many of them still remain and a lot of over-subtracted pixels newly appear as black points. They are typically stronger than diffuse $\mathrm{H}_{2}$ emission and still contribute as serious contaminations.

On the other hand, since the Galactic center is an extremely crowded region, not surprisingly a number of stellar continua are included in each spectral image. Typ-

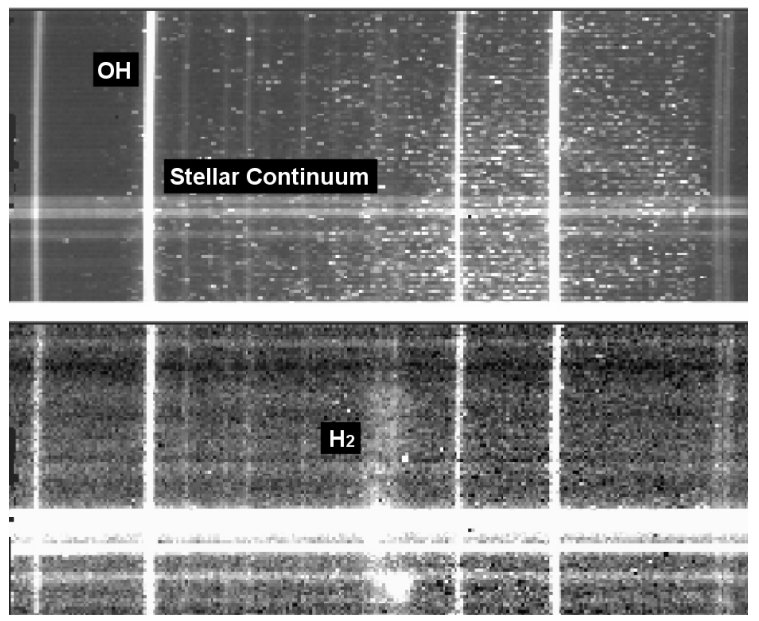

Fig. 2. Example of sky subtraction. (top) A raw spectral image before the sky subtraction. The horizontal axis is along spectral dispersion and the vertical axis is along spatial position on the slit. (bottom) After subtracting blank sky image, diffuse $\mathrm{H}_{2}$ emission becomes visible in the central part of the image. See the text for details.

ically two or three of them are much brighter than the $\mathrm{H}_{2}$ emission (Fig. 2). Therefore those contaminations should be cleaned up to obtain pure $\mathrm{H}_{2}$ spectra with high signalto-noise $(\mathrm{S} / \mathrm{N})$ ratios.

The residual bad pixels are corrected by using the IRAF package, NOAO.IMRED.CCDRED. COSMICRAYS, which detects cosmic rays or bad pixels in an image and replaces them with the average of neighboring pixels. We implement this task two times for each image. In the first run, bad pixels with positive values are corrected. Then, to take care of bad pixels with negative values, which are by-products of sky subtraction, we invert (multiply by -1) the once-corrected image and run COSMICRAYS again. By inverting the resulting image again to its original sign, the cleaning process for bad pixel completes (Fig. 3).

To remove continuum spectra of field stars, we use the IRAF package, NOAO.IMRED. GENERIC.BACKGROUND. Along each pixel row or column in an image, this task fits the background to a functional form and subtracts it. We apply linear fitting functions on both sides (at shorter and longer wavelengths) of the $\mathrm{H}_{2}$ emission line in each row in each image. As a result, stellar continua are subtracted successfully around the emission line (Fig. 4).

In order to clean up the residual telluric $\mathrm{OH}$ lines, we need a template image of $\mathrm{OH}$ lines which will be scaled to have identical intensity levels with the residual $\mathrm{OH}$ lines and then be subtracted from the object image. A blank sky image can be used as a template. However, it includes the dark and sky background which was already subtracted from the object image by the process of sky sub- 

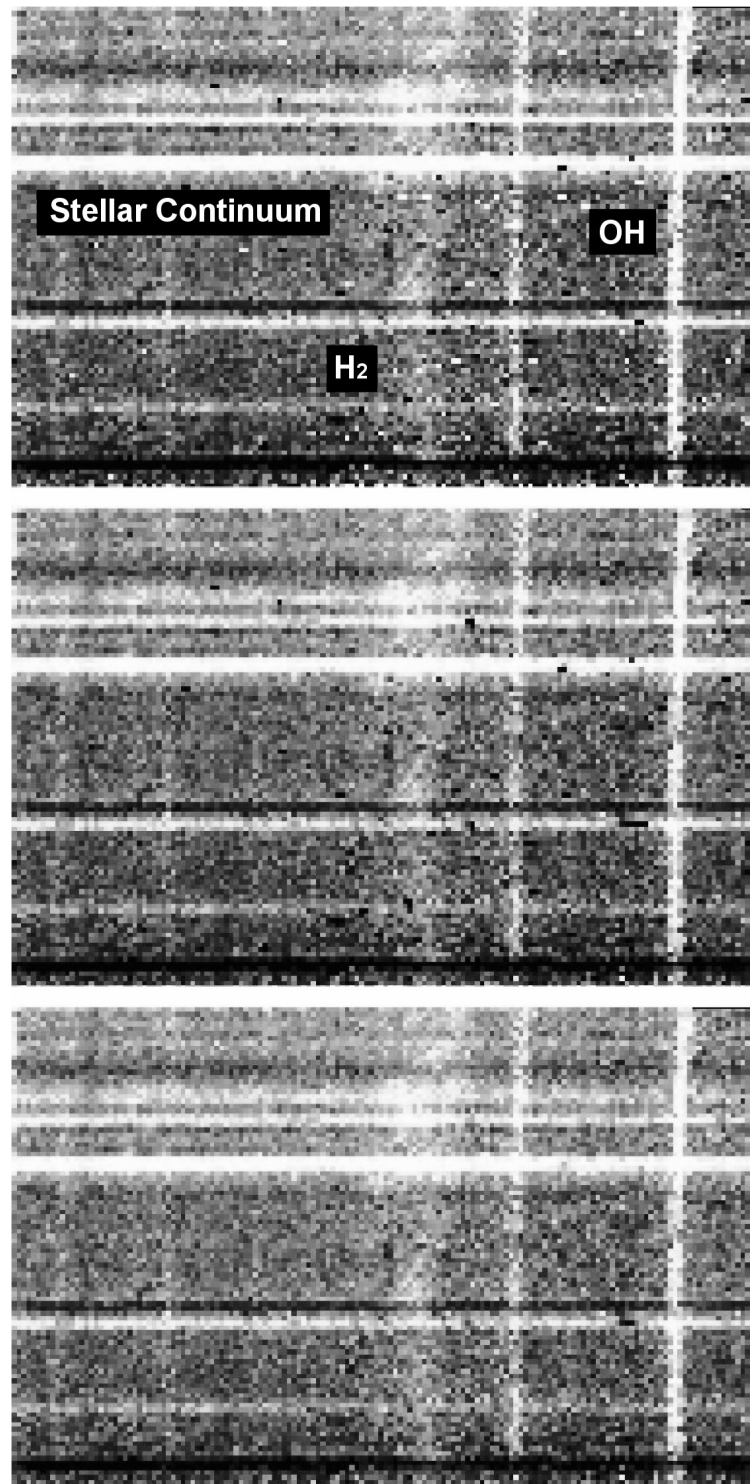

Fig. 3. Example of cleaning bad pixels. (top) A sky-subtracted spectral image. (middle) After the first correction for the bad pixels with positive values. (bottom) After the second correction for the negative bad pixels which were over-subtracted in the sky subtraction.

traction above. Thus, we make a template image of pure $\mathrm{OH}$ lines by subtracting a mode value of the background from the blank sky image. This template image, however, still contains contaminations. Therefore we clean up the template image (Fig. 5) before we use it to remove the residual $\mathrm{OH}$ lines in the object image (Fig. 6).

The sequence in the cleaning process (bad pixel - stellar continuum - telluric $\mathrm{OH}$ lines) is determined irreversibly. The correction for bad pixels should be done before removing $\mathrm{OH}$ lines, otherwise the bad pixels in the $\mathrm{OH}$ template will be added to the object image and we will
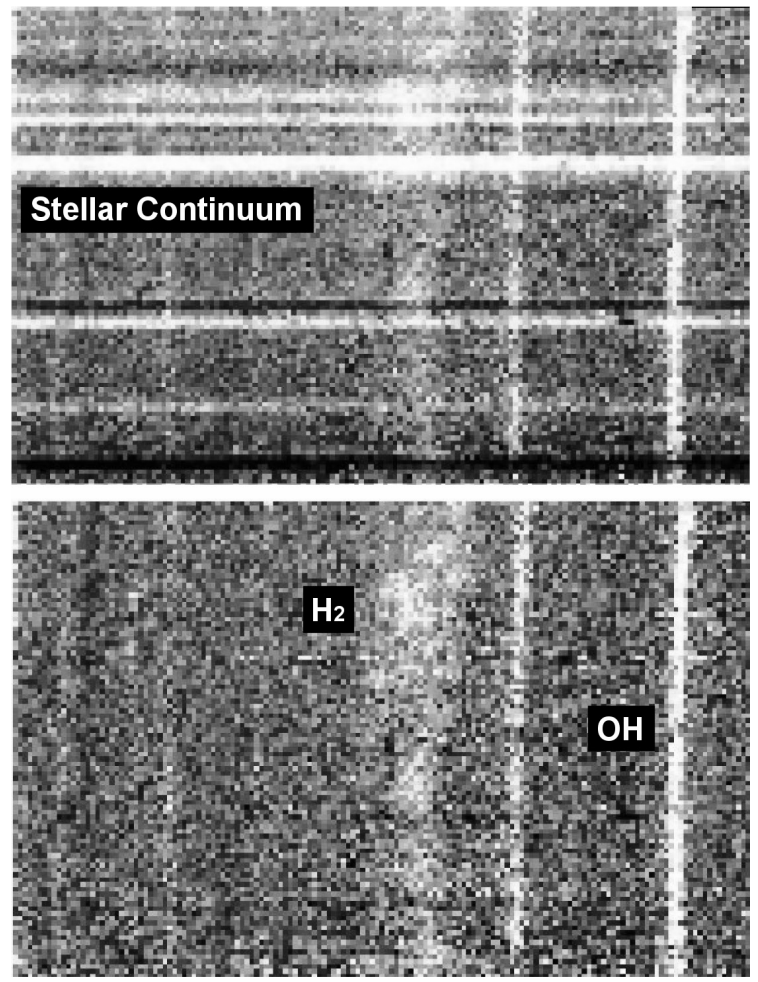

Fig. 4. Example of cleaning stellar continua. (top) The spectral image from Fig. 3 after the bad pixel correction. (bottom) After the correction for stellar continua.
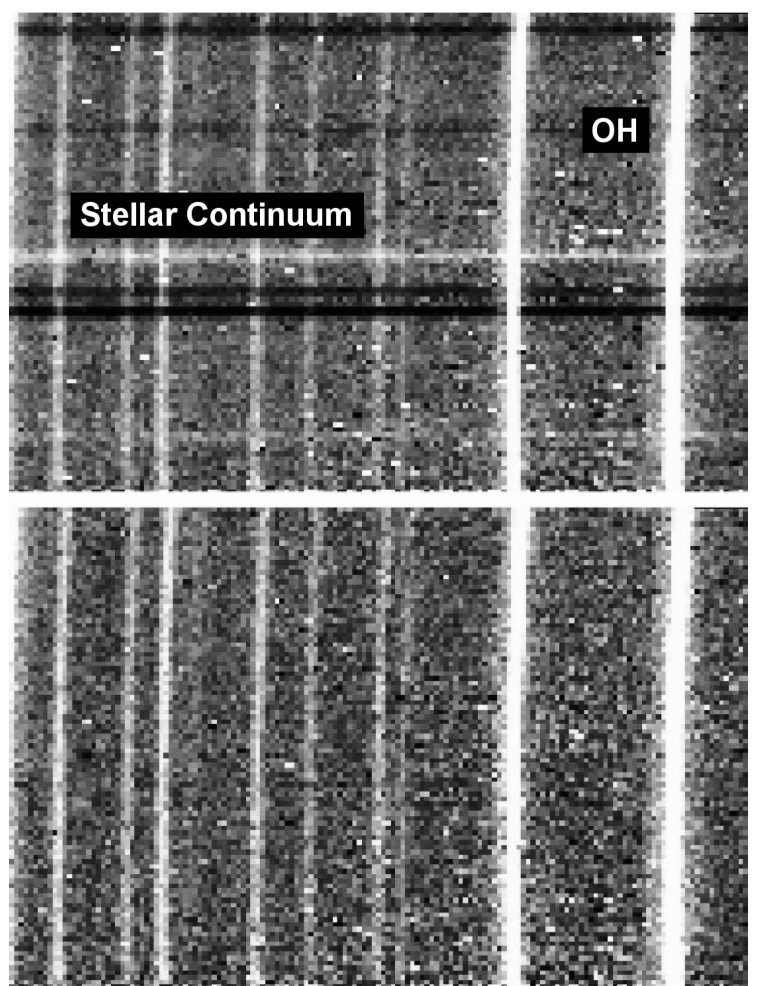

Fig. 5. Example of sky template for cleaning telluric $\mathrm{OH}$ lines. (top) $\mathrm{A}$ blank sky image. (bottom) A sky template image after correction for bad pixels and stellar continua. 

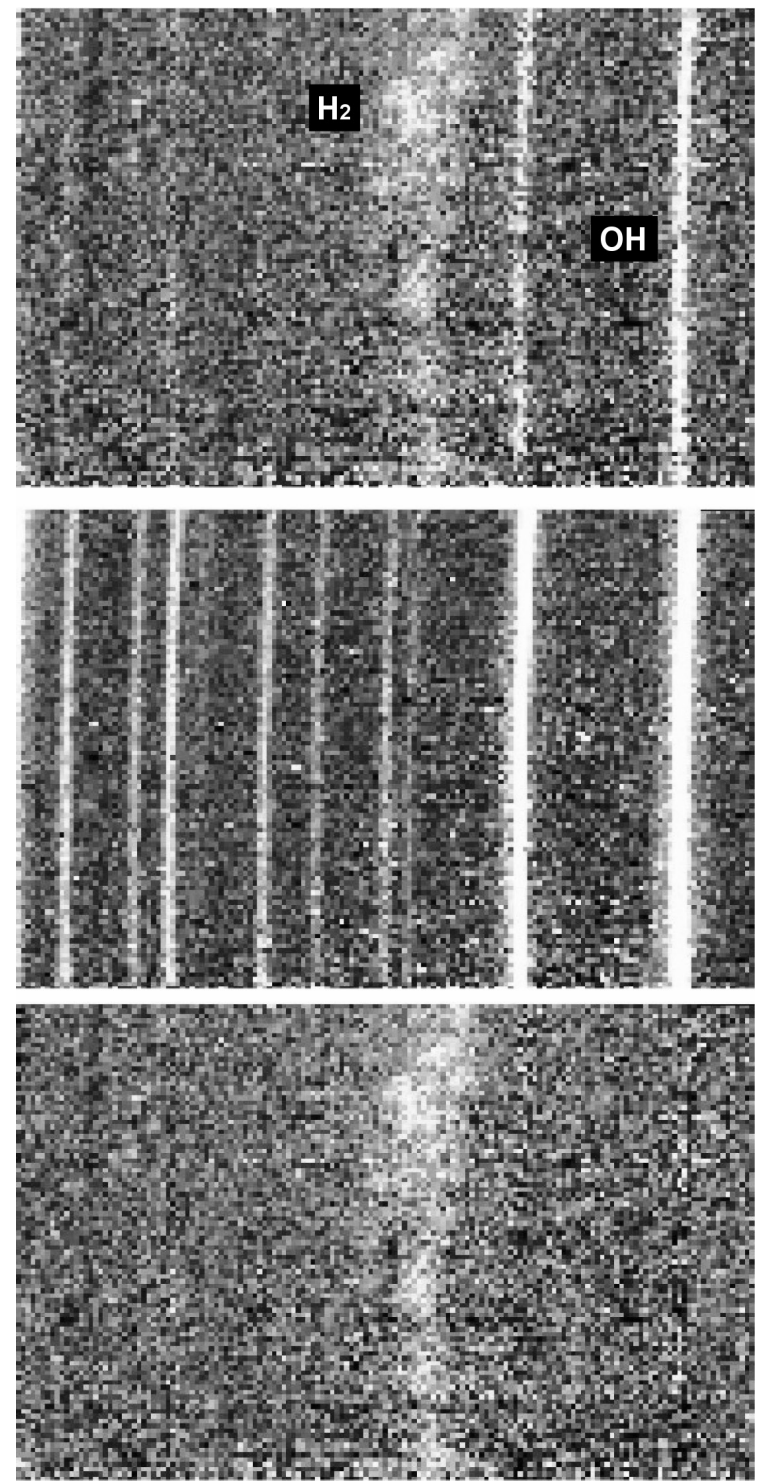

Fig. 6. Example of cleaning telluric $\mathrm{OH}$ lines. (top) The spectral image from Fig. 4 after the correction for bad pixels and stellar continua. (middle) The sky template of telluric $\mathrm{OH}$ lines from Fig. 5 after the correction for bad pixels and stellar continua. (bottom) After all the process of cleaning contaminations including the telluric $\mathrm{OH}$ lines.

lose or spoil a number of meaningful pixels. Also the stellar continuum subtraction should be done before $\mathrm{OH}$ correction to scale the template accurately to the object image. We found that it is better to correct bad pixels before subtracting continuum. It probably makes the continuum fit better. The cleaning process should be done before correcting spatial and spectral distortions since the distortion correction 'distort' original features in the images. Particularly it makes bad pixels larger and much more difficult to be identified and removed by COSMICRAYS.

\subsection{Correction for Spatial and Spectral Distor- tions and Wavelength Calibration}

To analyze the long slit spectrum, we should correct spectral distortion along the dispersion axis (i.e. curved stellar continuum) and spatial distortion along the slit (i.e. tilted or curved arc or telluric $\mathrm{OH}$ lines). In this stage of data reduction, IRAF tasks IDENTIFY, REIDENTIFY, FITCOORDS, and TRANSFORM in the package of NOAO. TWODSPEC.LONGSLIT are utilized. The correction for spectral distortion accompanies wavelength calibration as well.

For the correction of spectral distortion, we use spectrum of the standard star as a template. In principle, to fix distortions in the spectral direction, a standard star needs to be observed at different positions along the slit. However, since the spectral distortion of CGS4 in its echelle mode is not so serious (actually nearly negligible around the interested $\mathrm{H}_{2}$ line), stellar spectra obtained at two or three positions are enough to make a template. Typically, stars are jittered along the slit to subtract the sky background (by subtracting the jittered image from the original image). With one jittering, we get two templates (e.g. template 1 and template 2), each of which contains one stellar spectrum at the different position, respectively.

The procedure of spectral distortion correction is as follows. First, we use IDENTIFY to mark the position of the standard star spectrum across the central column in template 1. Second, we measure the position of the same standard spectrum across different columns in template 1 using REIDENTIFY. Then we repeat IDENTIFY and REIDENTIFY on template 2. Using the task FITCOORDS, we find a transformation solution for the distortion in the dispersion direction evident in these standard spectra. Finally, after verifying the solution using the standard star images first, we transform the object images using TRANSFORM.

In a very similar way we correct for distortions in the spatial direction. First, we run IDENTIFY on the sky $\mathrm{OH}$ spectra (across the central row this time) and input the wavelength of each $\mathrm{OH}$ line. We use four $\mathrm{OH}$ lines (at $2.1156116 \mu \mathrm{m}, 2.1176557 \mu \mathrm{m}, 2.1232424 \mu \mathrm{m}$, and $2.1249592 \mu \mathrm{m})$ for the $\mathrm{H}_{2}$ 1-0 S(1) spectral images. These telluric $\mathrm{OH}$ lines are bright enough for their pixel positions along the dispersion axis to be measured with a high accuracy and their wavelengths can be found in the literature (Rousselot et al. 2000). Next, we run REIDENTIFY, FITCOORDS, and TRANSFORM just like in the correction for spectral distortions. In Fig. 7, we show an example of the distortion correction. 

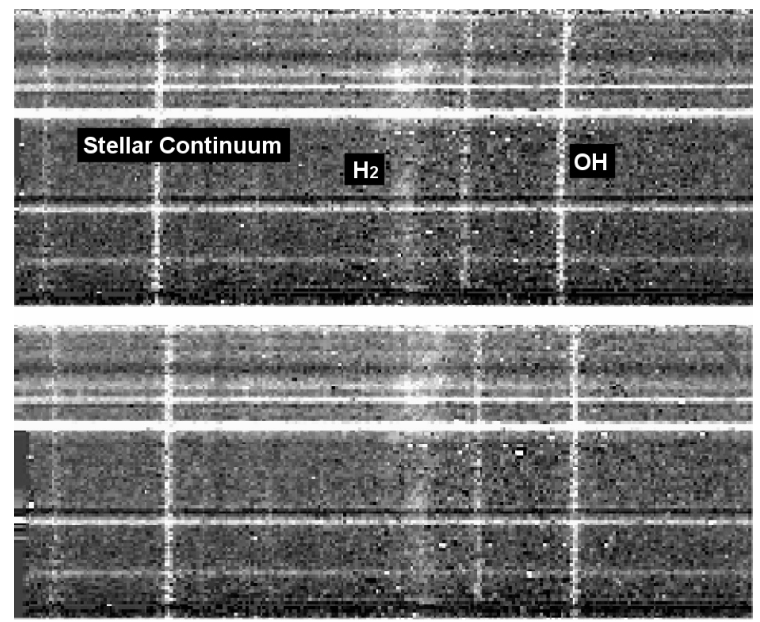

Fig. 7. Example of correction for spatial and spectral distortions. (top) A spectral image before the correction for distortions. (bottom) After correcting for both spatial and spectral distortions. Note that this is an example at the pre-cleaning stage which is still contaminated by stellar continua and telluric $\mathrm{OH}$ lines but shows best the impact of distortions.

We also calibrate wavelength of the data during the correction of spatial distortions, using known $\mathrm{OH}$ lines as references. The information of calibrated wavelength is written in FITS headers as coordinate along the axis of spectral dispersion. Then we can convert wavelength into velocity relative to the rest wavelength of $\mathrm{H}_{2}$ line using NOAO.ONEDSPEC.DISPTRANS. We can also correct for the motions of the Earth and Sun using ASTUTIL. RVCORRECT in order to determine local standard of rest (LSR) velocities.

The reliability of the wavelength calibration was verified by estimating its accuracy and precision. For accuracy, we measure calibrated wavelengths of all available $\mathrm{OH}$ lines in the sky images and compare them with known values (Rousselot et al. 2000). Then the average of the resulting differences is about $\pm 0.5 \mathrm{~km} \mathrm{~s}^{-1}$. For precision, we calculate dispersion of measured wavelengths for the $\mathrm{OH}$ line at $2.1232424 \mu \mathrm{m}$ which is nearest to the $\mathrm{H}_{2}$ 1-0 $S(1)$ line $(2.1218 \mu \mathrm{m})$. Then the resulting dispersion is as large as $\pm 1 \mathrm{~km} \mathrm{~s}^{-1}$.

Selected from the 2-D spectral images we observed in the northern part of Sgr A East, several raw (only sky-subtracted) images and their reduced results are compared with each other in Fig. 8.
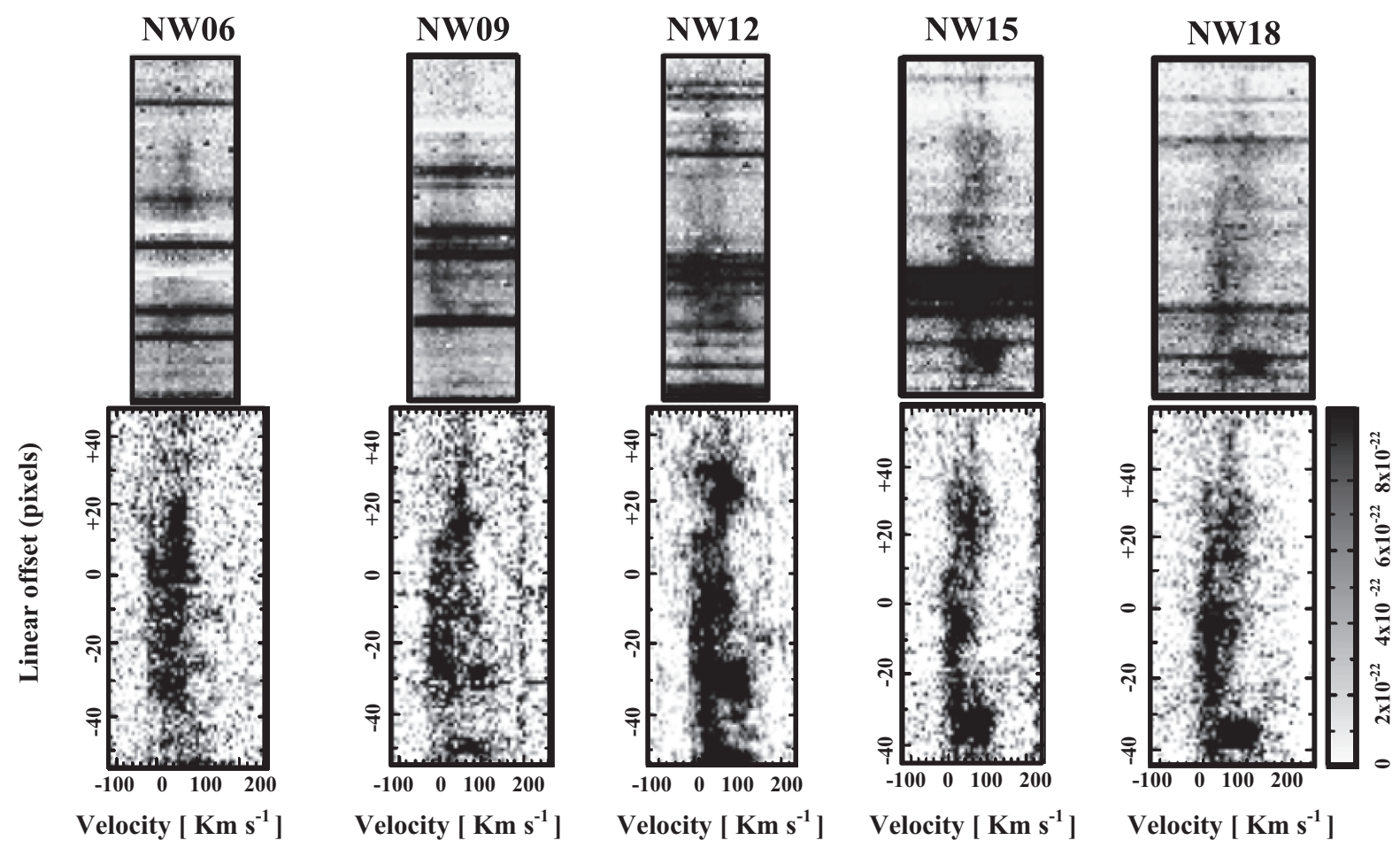

Fig. 8. Results of data reduction for sample 2D spectral images. (top) Raw (only sky-subtracted) spectral images in negative. Slit position ID is indicated on top of each image. (bottom) After reduction and calibrations. Vertical axis along the slit represents offset from the reference position in pixels. Horizontal axis along the spectral dispersion represents LSR velocity relative to the rest wavelength of $\mathrm{H}_{2}$ 1-0 S(1) line in $\mathrm{km} \mathrm{s}^{-1}$. The intensity scale runs from 0 to $10^{-21} \mathrm{Wm}^{-2} \operatorname{arcsec}^{-2} \mathrm{~km}^{-1} \mathrm{~s}$. 


\section{THREE-DIMENSIONAL DATA REDUCTION}

By unifying the high resolution 2D spectral images into a $3 \mathrm{D}$ data cube, we can investigate the structure and kinematics of the $\mathrm{H}_{2}$ emitting gas simultaneously. At the wavelengths shorter than IR, 3D observations with such high spectral resolutions using echelle grating as in our case have not been so popular. However, in radio observations, it is not unusual to map a large area with a very high resolution in frequency. Thus there are some useful tools for reducing and handling 3D data.

MIRIAD (Sault et al. 1995, Hoffman et al. 1996) is a radio interferometry data reduction package, in the user environment with a large set of moderate-sized programs which perform individual tasks, involving calibration, mapping, deconvolution and image analysis of interferometric data. However, MIRIAD can also be used for general reduction of continuum and spectral line data in various formats (including FITS) throughout all stages of image analysis and output. Hence we choose MIRIAD for our near-IR 3D data reduction.

First, we have to transform the FITS data reduced by IRAF into the MIRIAD data format. This transformation of data format is easily achieved by the MIRIAD task FITS. This task also translates some standard information contained in the header of the FITS files into a MIRIADreadable format (called 'item'). However, the FITS task cannot understand all the 'fields' in the original FITS header since the definitions are often different between

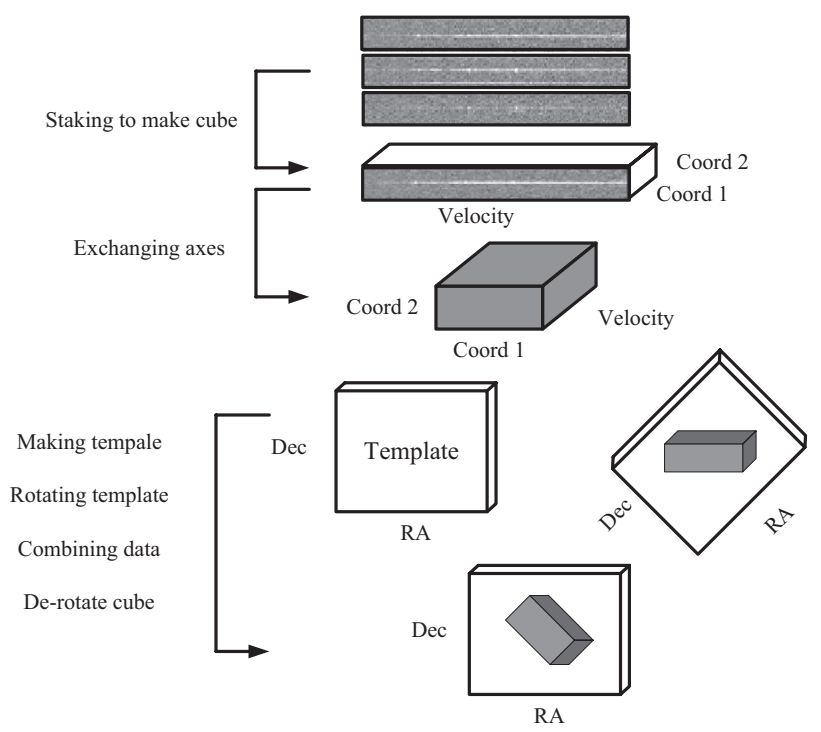

Fig. 9. Sequence of making a data cube (see text for the details). Note that coord 1 and coord 2 in the first half of the process is not celestial coordinates but with respect to the slit axes; coord 2 is along the slit length and coord1 is along the direction of the slit-scanning. observatories or instruments. Thus we need to manually translate some necessary FITS fields into MIRIAD items.

Then we use the MIRIAD task IMCAT to stack the 2D spectral images into a single 3D data unit (or data cube). We build data cubes separately for individual slit-scanning group since spectral images in each group are all the same in dimension and share a common position reference and slit angle. As a result, 11 data cubes with different dimensions, positions, and inclination angles are built from the entire survey area.

However, in order to investigate the large-scale structure in the surveyed region, we need to combine the unit data cubes into a single and global data cube. For this purpose, every pixel in each unit data cube should know its exact position on the sky; i.e. we need to set coordinate information on each data cube. The sequence of this process is presented in Fig. 9. As seen in the figure, the simply stacked data cubes have two spatial axes; one along the slit length and the other along the slitscanning direction. If the slit position angle is simply a multiple of right angle (i.e. $0^{\circ}, 90^{\circ}, 180^{\circ}$, and $270^{\circ}$ ), those two axes could be directly projected on Right Ascension (R.A.) and Declination (Dec), respectively, or vice versa. However, in our observations with various slit position angles, this coordinating process becomes slightly complicated. First, we make a template cube which contains no data value but is defined in celestial coordinates (R.A. and Dec) along two spatial axes. Second, we rotate the template by the slit position angle. Third, we merge the data cube with this rotated template cube. Finally, we derotate the merged cube so that each data pixel is located at its exact position on the sky.

Before the coordinating process above, we should resample the data cube along all its three axes using the MIRIAD task REGRID. The original pixel scale in the data cube, which is determined by the mapping resolution of the slit scanning, is not in a square shape but $0.9 " \times 3$ " along the slit length and perpendicular to it, respectively. Thus the intensity distribution could become biased after rotation of the cube. To minimize this bias effect, we divide each pixel into much smaller, square, pixels with a scale of $0.3 " \times 0.3$ " (Fig. 10). This higher sampling resolution has another merit of more accurate matching when we combine the individual data cubes into the global cube. For this unification, we also re-sample and unify the velocity axes of the data cubes into a common definition (68 pixels with a scale of $7.3 \mathrm{~km} \mathrm{~s}^{-1}$ in the range from -200 to $+300 \mathrm{~km} \mathrm{~s}^{-1}$ ).

MIRIAD has several tasks for mosaicking data cubes. Among them LINMOS is the most appropriate task for 

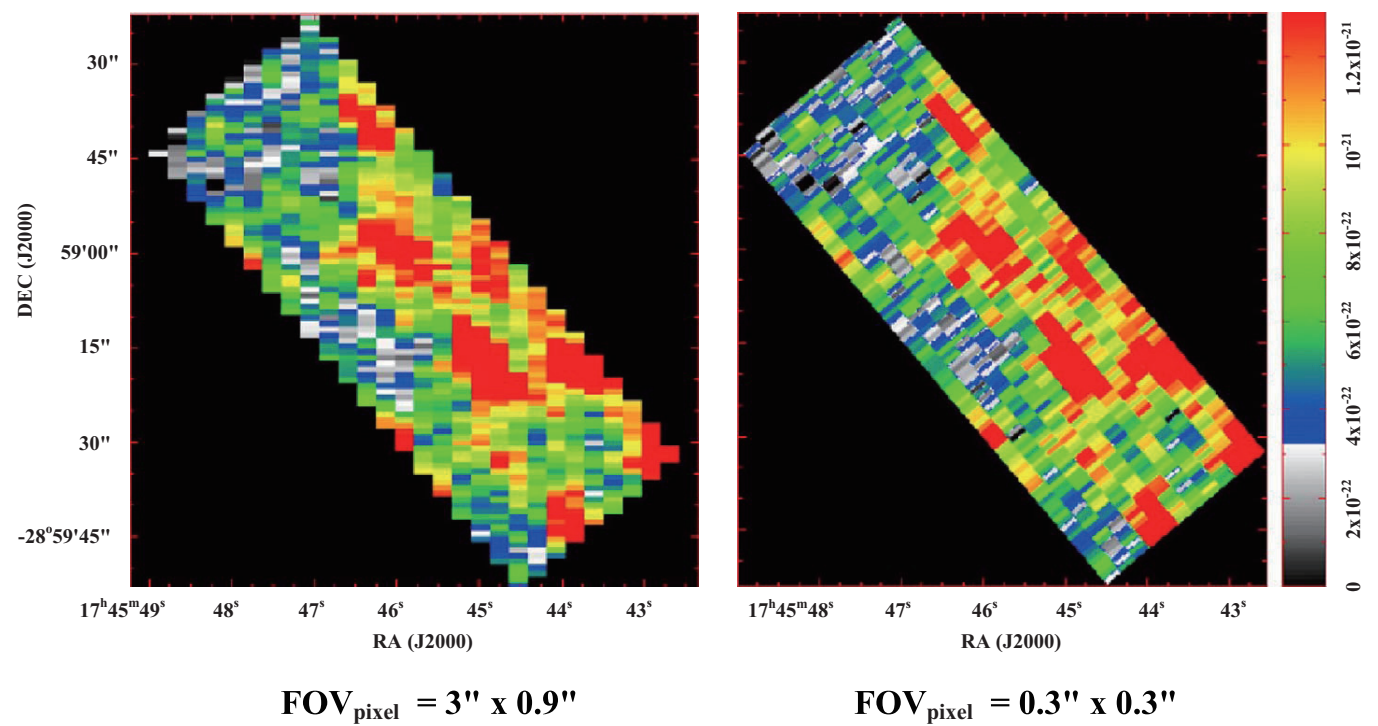

$\mathrm{FOV}_{\text {pixel }}=0.3^{\prime \prime} \times 0.3^{\prime \prime}$

Fig. 10. Example of re-sampling a data cube (velocity-averaged $\mathrm{H}_{2} 1-0 \mathrm{~S}(1)$ map). The intensity level is indicated by the right-side bar from 0 to $1.3 \times 10^{-21} \mathrm{Wm}^{-2} \operatorname{arcsec}^{-2} \mathrm{~km}^{-1} \mathrm{~s}$.

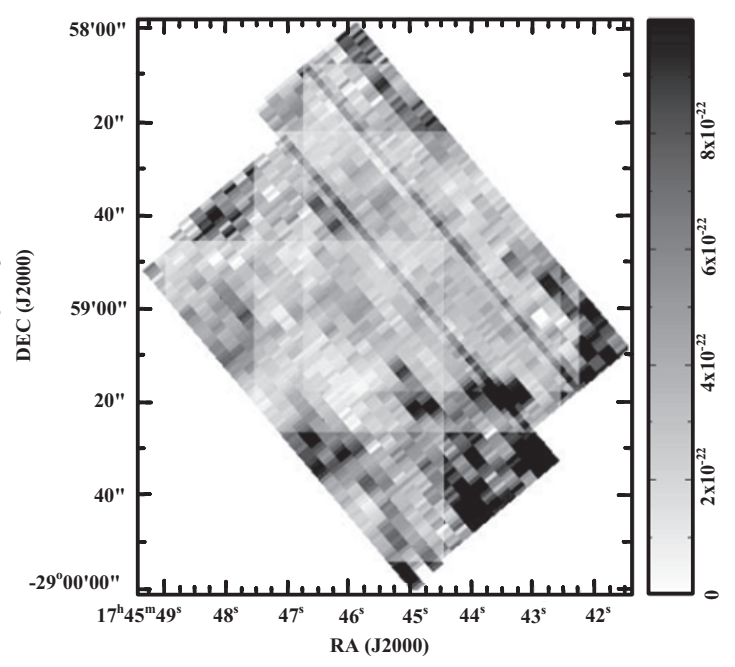

Fig. 11. Unsuccessful example of mosaicking data cubes using the MIRIAD task LINMOS. The velocity-averaged $\mathrm{H}_{2}$ 1-0 S(1) map from such a combined data cube. The intensity level is indicated by the right-side bar in $\mathrm{Wm}^{-2} \operatorname{arcsec}^{-2} \mathrm{~km}^{-1} \mathrm{~s}$.

non-radio type data like ours. However, while using LINMOS for mosaicking data cubes, we found problems in overlapped regions (Fig. 11). Since the pixel values in the overlapped region are averaged by LINMOS, when an observed position in one cube is overlapped by a blank position in another cube, the averaged pixel value in the combined cube becomes only a half of what should be. The blank regions are byproducts from the cube rotation, but MIRIAD regards the pixel values in those blanks as meaningful zero.

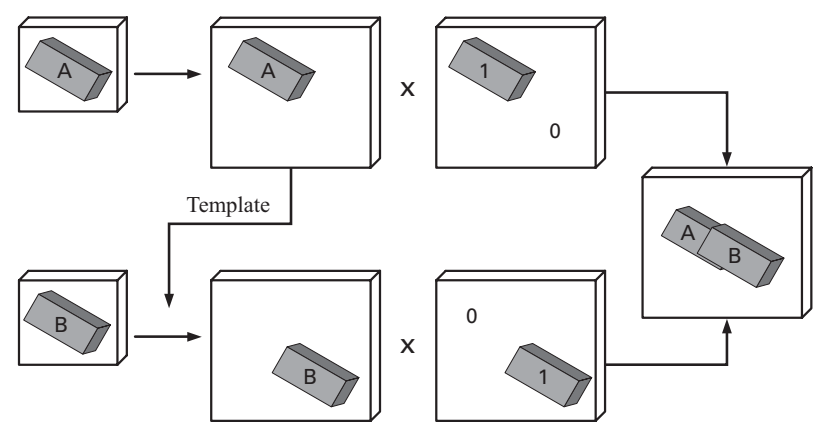

Fig. 12. Schematic diagram of weighted mosaicking. Note that the extended cube $A$ is used as a template when extending cube B for two cubes to have the same dimension and format.

Thus we implement a 'weighted mosaic' for our data cubes;

$$
\operatorname{cube}_{\mathrm{A}}+\operatorname{cube}_{\mathrm{B}}=\frac{\text { cube }_{\mathrm{A}^{\prime}} \times \operatorname{cube}_{\mathrm{A}, \mathrm{W}}+\operatorname{cube}_{\mathrm{B}^{\prime}} \times \text { cube }_{\mathrm{B}, \mathrm{W}}}{\text { cube }_{\mathrm{A}, \mathrm{W}}+\operatorname{cube}_{\mathrm{B}, \mathrm{W}}}
$$

when cube $e_{A, W}>0$ or cube $e_{B, W}>0$. Here the operation is done pixel by pixel. cube $\mathrm{A}^{\prime}$ and cube $\mathrm{B}^{\prime}$ are extended cubes from the original data cubes to a volume enough for the combined cube. cube ${ }_{\mathrm{A}, \mathrm{W}}$ and cube $\mathrm{B}_{\mathrm{W}, \mathrm{W}}$ are template cubes containing the weighting information (Fig. 12).

We present results from the mosaicking process in Fig. 13. Throughout all the following stages thereafter for scientific discussions in Lee et al. (2008), we only need this unified cube for the entire region for all kind of analyses such as extracting spectrum, velocity channel maps, 


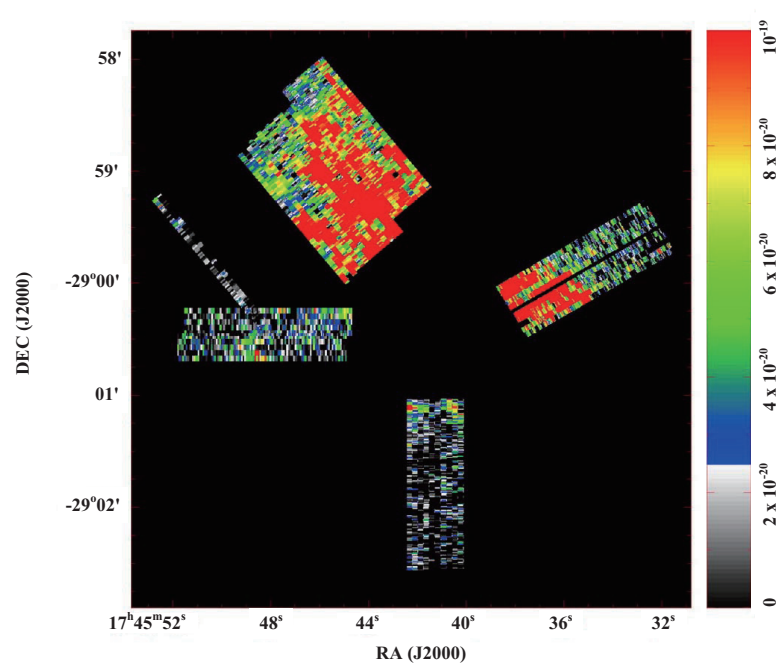

Fig. 13. The velocity-integrated $\mathrm{H}_{2}$ 1-0 S(1) map of the entire surveyed region from the combined data cube. The intensity level is indicated by the right-side bar in units of $\mathrm{Wm}^{-2} \operatorname{arcsec}^{-2} \mathrm{~km}^{-1} \mathrm{~s}$.

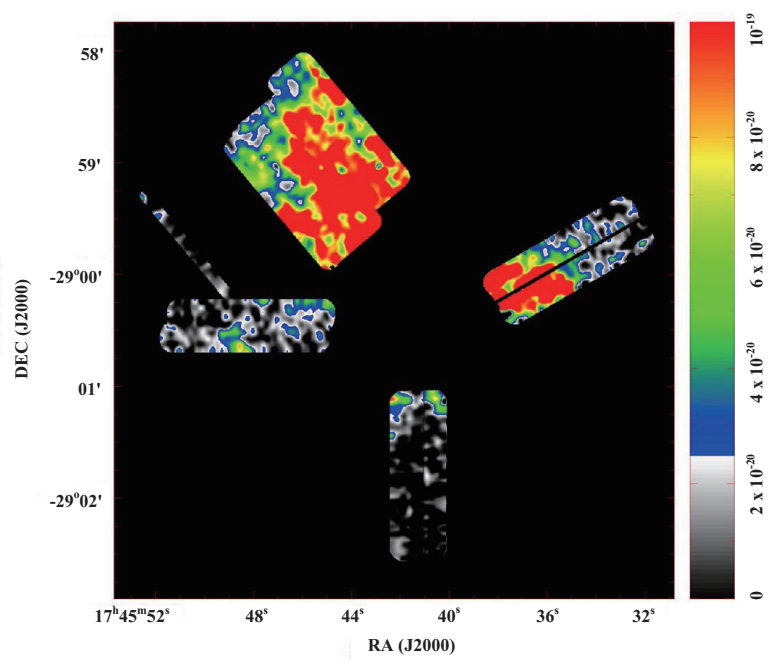

Fig. 14. The velocity-integrated $\mathrm{H}_{2}$ 1-0 S(1) map of the entire surveyed region: smoothed by 3 "-FWHM. The intensity level is indicated by the right-side bar in units of $\mathrm{Wm}^{-2} \operatorname{arcsec}^{-2} \mathrm{~km}^{-1} \mathrm{~s}$.

position-velocity diagrams, and direct comparison with radio data. A smoothed version of the global cube, which is convolved with a Gaussian of FWHM = 3 arcsec and has a higher $\mathrm{S} / \mathrm{N}$ ratio, is also presented in Fig. 14.

\section{SUMMARY}

Integral field spectroscopy is a powerful observational technic providing higher efficiency and accuracy. In addition to the ability of getting 3D information over the field of view in a single exposure, no light loss through a slit or an aperture keeps the highest throughput of the instrument. The simultaneously obtained 3D data enables reliable calibration both over the field of view and over the wavelength range since there is no time variation between data points in the cube.

Applications using integral field spectrographs have been mostly limited to observations of low dispersion spectrum from small targets such as interacting galaxies. To cover a large field of view with decently high spatial and spectral resolutions, a very long pseudo-slit optics and a very large detector, which are technically difficult and not cost effective, are needed. Scanning observations using long-slit echelle spectrographs are practical choice for this kind of applications although they are less efficient in observing time and less reliable for calibration between slit exposures.

Three-dimensional data, especially at a high velocity resolution, require new methods for data reduction and analysis. In this paper, we introduced one practical approach utilizing a well established tool, MIRIAD, which has been used for years in the field of radio astronomy and is equipped with a number of powerful functions for exploring the spatial-velocity space of 3D data. Our sample application is a survey for a large region with various configurations in the slit position and angle, which must be a complex enough case to test the general capability of the tool. The flexibility and generality of MIRIAD enables us to organize and handle such complicated data cubes. This type of 3D survey would be useful for studies on dynamical structure of large extended objects like supernova remnants and planetary nebulae.

First half of this paper is dedicated to a guideline on the data reduction for long-slit IR spectroscopy. To recover diffuse emission features of extended objects from such contaminations as background radiation, bad pixels, telluric $\mathrm{OH}$ lines, and stellar continuum spectra, careful implementation of image processing in a proper sequence is required. We suggested a number of IRAF tools for this process as well as some spectroscopy and geometry tools for image-distortion correction and wavelength calibration.

\section{ACKNOWLEDGEMENTS}

Helps of Chris Davis at the Joint Astronomy Centre and Tom Geballe at the Gemini Observatory for the observation and data reduction were essential for our study. Particularly, we followed the reduction guide for CGS4 data 
by Chris Davis. We also thank the staff at UKIRT for their excellent support during the observation. The United Kingdom Infrared Telescope is operated by the Joint Astronomy Centre on behalf of the Science and Technology Facilities Council of the U.K. S.P. was supported by WCU program through NRF funded by MEST of Korea (R3110016). M.C. was supported by the International Research \& Development Program of the National Research Foundation of Korea (NRF) funded by the Ministry of Education, Science and Technology (MEST) of Korea (grant number: K20901001400-09B1300-03210).

\section{REFERENCES}

Burton, M. G. \& Allen, D. A. 1992, Proc. Ast. Soc. Aust., 10, 55 Eisenhauer, F., Abuter, R., Bickert, K., Biancat-Marchet, F., Bonnet, H., Brynnel, J., Conzelmann, R. D., Delabre, B., Donaldson, R., Farinato, J., Fedrigo, E., Genzel, R., Hubin, N. N., Iserlohe, C., Kasper, M. E., Kissler-Patig, M., Monnet, G. J., Roehrle, C., Schreiber, J., Stroebele, S., Tecza, M., Thatte, N. A., \& Weisz, H. 2003, SPIE Proc., 4841, 1548

Hoffman, W., Hudson, J., Sharpe, R. K., Grossman, A. W., Morgan, J. A., \& Teuben, P. J. 1996, ASPC, 101, 436

Larkin, J., Barczys, M., Krabbe, A., Adkins, S., Aliado, T., Amico, P., Brims, G., Campbell, R., Canfield, J., Gasaway, T., Honey, A., Iserlohe, C., Johnson, C., Kress, E., LaFreniere, D., Lyke, J., Magnone, K., Magnone, N., McElwain, M., Moon, J., Quirrenbach, A., Skulason, G., Song, I., Spencer, M., Weiss, J., \& Wright, S. 2006, SPIE Proc., 6269, 62691A

Lee, S. \& Pak, S. 2006, JKAS, 39, 151

Lee, S., Pak, S., Choi, M., Davis, C. J., Geballe, T. R., Herrn- stein, R. M., Ho, P. T. P., Minh, Y. C., \& Lee, S. G. 2008, ApJ, 674, 247, doi: 10.1086/524128

Lee, S., Pak, S., Davis, C. J., Herrnstein, R. M., Geballe, T. R., Ho, P. T. P., \& Wheeler, J. C. 2003, MNRAS, 341, 509, doi: 10.1046/j.1365-8711.2003.06425.x

Lee, S., Pak, S., Lee, S. G., Davis, C. J., Kaufman, M. J., Mochizuki, K., \& Jaffe, D. T. 2005, MNRAS, 361, 1273, doi: 10.1111/j.1365-2966.2005.09259.x

Lee, S., Yuk, I. S., Lee, H., Wang, W., Park, C., Park, K. J., Chun, M. Y., Pak, S., Strubhar, J., Deen, C., Gully-Santiago, M., Rand, J., Seo, H., Kwon, J., Oh, H., Barnes, S., Lacy, J., Goertz, J., Park, W. K., Pyo, T. S., \& Jaffe, D. T. 2010, SPIE Proc., 7735, 77352K

McGregor, P., Hart, J., Conroy, P., Pfitzner, L., Bloxham, G., Jones, D., Downing, M., Dawson, M., Young, P., Jarnyk, M., \& van Harmelen, J. 2003, SPIE Proc., 4841, 1581

McLean, I. 1997, Electronic Imaging in Astronomy: Detectors and Instrumentation (Chichester: Praxis Publishing Ltd.), p.199

Mountain, C. M., Robertson, D. J., Lee, T. J., \& Wade, R. 1990, SPIE Proc., 1235, 25

Rousselot, P., Lidman, C., Cuby, J. G., Moreels, G., \& Monnet, G. 2000, A\&A, 354, 1134

Sault, R. J., Teuben, P. J., \& Wright, M. C. H. 1995, ASPC, 77, 433

Weitzel, L., Krabbe, A., Kroker, H., Thatte, N., Tacconi-Garman, L. E., Cameron, M., \& Genzel, R. 1996, A\&AS, 119, 531

Yuk, I. S., Jaffe, D. T., Barnes, S., Chun, M. Y., Park, C., Lee, S., Lee, H., Wang, W., Park, K. J., Pak, S., Strubhar, J., Deen, C., Oh, H., Seo, H., Pyo, T.-S., Park, W. K., Lacy, J., Goertz, J., Rand, J., \& Gully-Santiago, M. 2010, SPIE Proc., 7735, $77351 \mathrm{M}$ 